\title{
ÚNG DỤNG PHƯƠNG PHÁP BÌNH SAI TỬ DO ĐỂ XỬ LÝ SỐ LIỆU LƯớI MẠTT BẰNG THI CÔNG CÔNG TRÌNH
}

\author{
NGUYẼ̃N HÀ, TRÀN THUỲ LINH \\ Trường Đại học Mỏ Địa chất
}

\section{Tóm tắt:}

Bài báo trình bày kết quả nghiên cứu ứng dụng phương pháp bình sai tự do để xử lý số liệu các mạng lưới thi công công trình. Đã đề xuất thuật toán và quy trình tính phù hợp với dạng lưới nêu trên. Khảo sát lý thuyết và các kết quả ưng dụng ở thực tế sản xuất cho thấy tính ưu việt của phương pháp bình sai tự do trong xử lý số liệu nhiều dạng lưới mặt bằng thi công công trình.

\section{1. Đặt vấn đề}

Lưới khống chế trắc địa công trình là những mạng lưới được thành lập trong quá trình khảo sát, thiết kế, thi công và vận hành các công trình kỹ thuật thuộc nhiều lĩnh vực kinh tế-xã hội khác nhau. Theo mục đích sử dụng, lưới trắc địa công trình được chia thành 3 nhóm, gồm lưới khảo sát, lưới thi công và lưới quan trắc chuyển dịch biến dạng. Yêu cầu độ chính xác, mật độ điểm và một số chỉ tiêu kỹ thuật khác của lưới trắc địa công trình có nhiều điểm khác biệt so với các mạng lưới trắc địa Nhà nước do loại lưới này cần phải đáp ứng những yêu cầu đa dạng của các giai đoạn xây dựng và của từng loại công trình.

Tuy nhiên, điểm đặc trưng nhất của lưới trắc địa công trình là các mạng lưới được thành lập trong hệ tọa độ cục bộ với yêu cầu độ chính xác tăng dần từ bậc trước đến bậc sau (lưới thi công), hoặc là dạng lưới đo lặp với mục đích xác định độ chuyển dịch các điểm mốc trong lưới (lưới quan trắc biến dạng công trình). Như vậy, lưới khống chế trắc địa công trình thuộc về dạng lưới trắc địa tự do và do đó, trong tính toán xử lý số liệu lưới áp dụng phương pháp bình sai tự do là phù hợp nhất.

\section{Quy trình tính toán và những tính chất của phương pháp bình sai tự do}

Mô hình bài toán bình sai tự do có thể được thể hiện dưới dạng bình sai gián tiếp kèm điều kiện với nội dung như sau $[1,3]$ :

Trong mạng lưới tự do chọn ẩn số là tọa độ (đối với lưới mặt bằng) hoặc độ cao (đối lưới lưới thủy chuẩn) của tất cả các điểm, khi đó các bước tính toán sẽ được thực hiện theo trình tự:

1. Thành lập hệ phương trình số hiệu chỉnh $\quad A \delta X+L=V$

trong đó: $\mathrm{A}$ là ma trận hệ số, $\delta \mathrm{X}$ là vector ẩn số, $V$ và $L$ là các vector số hiệu chỉnh và số hạng tự do.

Do trong lưới thiếu các yếu tố định vị tối thiểu nên ma trận hệ số hệ phương trình số hiệu chỉnh (1) có các cột phụ thuộc (số cột phụ thuộc bằng với số lượng số liệu gốc còn thiếu và được gọi là số khuyết của lưới).

2. Kết quả chuyển từ hệ phương trình số hiệu chỉnh sang hệ phương trình chuẩn theo nguyên lý số bình phương nhỏ nhất sẽ thu được: $R \delta X+b=0$

$$
\text { với: } \quad R=A^{T} P A ; \quad b=A^{T} P L
$$


3. Hệ phương trình (2) có vô số nghiệm, một vector nghiệm riêng của hệ phương trình trên được xác định thông qua hệ điều kiện ràng buộc đối với vector ẩn số, dạng:

$$
C^{T} \delta X=0
$$

4. Kết hợp các biểu thức (2) và (3) sẽ thu được hệ phương trình chuẩn mở rộng:

$$
\left[\begin{array}{cc}
R & C \\
C^{T} & 0
\end{array}\right] \times\left[\begin{array}{c}
\delta x \\
K
\end{array}\right]+\left[\begin{array}{l}
b \\
0
\end{array}\right]=0
$$

Khi đó nghiệm của hệ phương trình (4) sẽ là:

$$
\delta X=-R^{\sim} b
$$

Với ma trận giả nghịch đảo $\mathrm{R}^{\sim}$ được tính theo công thức:

$$
R^{\sim}=\left(R+C P_{0} C^{T}\right)^{-1}-B\left(C^{T} B\right)^{-1}\left(B\left(C^{T} B\right)^{-1}\right)^{T}
$$

Phân tích thuật toán bình sai lưới tự do nêu trên sẽ rút ra được kết luận về sự khác biệt cơ bản trong nguyên tắc xử lý giữa phương pháp bình sai lưới tự do và bình sai lưới phụ thuộc:

1-Trong lưới phụ thuộc tập hợp trị đo và tọa độ các điểm gốc trong lưới là đủ để xác định vector tọa độ bình sai duy nhất.

2-Trong bình sai lưới tự do, tập hợp trị đo và tọa độ của các điểm trong lưới mới chỉ xác định được cấu trúc nội tại của lưới. Để tìm được nghiệm riêng cần phải bổ sung vào dữ kiện của bài toán một số điều kiện ràng buộc đối với vector ẩn số.

Kết quả bình sai tự do có nhiều tính chất khác biệt so với các phương pháp xử lý lưới thông thường, trong số đó 2 tính chất sau là rất đáng chú ý sau [2]:

1- Vector trị bình sai của các đại lượng đo là duy nhất, không phụ thuộc vào sự lựa chọn ma trận định vị $C$ cũng như lựa chọn vector tọa độ (độ cao) gần đúng, có nghĩa là các vector tọa độ (độ cao) bình sai trong lưới tự do có sự đồng dạng hình học đối với mọi phương án lựa chọn ma trận $C$ và lựa chọn vector tọa độ (độ cao) gần đúng.
2- Hai vector tọa độ (độ cao) bình sai ứng với 2 lựa chọn điều kiện định vị $C$ và tọa độ gần đúng khác nhau có mối liên hệ thông qua biểu thức:

$$
X_{2}=X_{1}+T C^{T}\left(X_{2}{ }^{(0)}-X_{1}{ }^{(0)}\right)
$$

Điều này dẫn đến hiệu quả là vector tọa độ (độ cao) bình sai phụ thuộc vào tọa độ (độ cao) các điểm có $C$ à không phụ thuộc vào tọa độ (độ cao) của các điểm có $\mathrm{C}=0$ do đó, có thể coi các điểm có $C$ cà là điểm định vị lưới.

Như vậy, việc xác định ma trận $C$ sẽ ảnh hưởng trực tiếp đến quá trình định vị lưới và có ý nghĩa rất quan trọng đối với kết quả bình sai. Do tính chất đa dạng và những tiêu chuẩn, yêu cầu kỹ thuật rất chặt chẽ được đề ra đối với các mạng lưới trắc địa công trình nên việc xác định biện pháp xử lý, tiêu chuẩn định vị của mỗi loại lưới cũng khác nhau. Dang của ma trận $C$ phụ thuộc vào chủng loại, số khuyết của lưới. Phần tiếp theo dưới đây chúng tôi sẽ phân tích cụ thể hơn vấn đề định vị lưới mặt bằng và độ cao tự do trong trắc địa công trình.

Đối với lưới khống chế mặt bằng, ma trận $C$ được chọn theo yêu cầu của việc định vị tối ưu lưới, có 2 khả năng: hoặc là định vị tối ưu theo độ lệch vị trí điểm hoặc là tối ưu theo độ lệch hướng [2].

Giả sử $N$ và N' biểu thị vị trí trước và sau bình sai của một điểm, khi đó khoảng cách $V_{i}=N N^{\prime}$ được gọi là độ lệch vị trí điểm, hình chiếu của véc tơ $V_{\mathrm{i}}$ theo hướng thẳng góc với phương vị $\alpha_{i}$ được gọi là độ lệch theo hướng $\alpha_{i}$.

Định vị lưới theo độ lệch vị trí điểm được thực hiện theo nguyên tắc "Tổng bình phương độ lệch vị trí các điểm định vị là nhỏ nhất". Cụ thể, độ lệch tọa độ của $\mathrm{K}$ điểm định vị trong lưới phải thỏa mãn biểu thức:

$$
\sum_{i=1}^{K} V_{i}^{2}=\sum_{i=1}^{K}\left(V x_{i}^{2}+V y_{i}^{2}\right)=\min
$$


Để định vị lưới mặt bằng theo độ lệch vị trí điểm, ma trận định vị $C$ đối với từng điểm định vị được xác định theo công thức

$$
C_{i}=\left[\begin{array}{cccc}
1 & 0 & Y_{i} & X_{i} \\
0 & 1 & -X_{i} & Y_{i}
\end{array}\right]
$$

Định vị lưới theo độ lệch hướng dựa trên nguyên tắc: "Tổng bình phương độ lệch hướng của các điểm định vị là nhỏ nhất". Khi đó điều kiện định vị tối ưu sẽ là:

$$
\sum_{i=1}^{K} V \alpha_{i}^{2}=\min
$$

Để định vị lưới tối ưu theo hướng cần chọn ma trận định vị $\mathrm{C}$ theo công thức:

$$
C_{i}=\left[\begin{array}{cc}
\sin ^{2} a_{i} & -\sin \alpha_{i} \cos \alpha_{i} \\
-\sin \alpha_{i} \cos \alpha_{i} & \cos ^{2} \alpha_{i} \\
A_{\alpha i} \sin \alpha_{i} & A_{m i} \sin \alpha_{i} \\
-A_{\alpha i} \cos \alpha_{i} & -A_{m i} \cos \alpha_{i}
\end{array}\right]
$$

với: $\quad A_{\alpha_{i}}=X_{i} \operatorname{Cos}\left(\alpha_{i}\right)+Y_{i} \operatorname{Sin}\left(\alpha_{i}\right)$

$$
A_{m_{i}}=X_{i} \operatorname{Sin}\left(\alpha_{i}\right)-Y_{i} \operatorname{Cos}\left(\alpha_{i}\right)
$$

\section{Xử lý số liệu lưới khống chế thi công mặt bằng}

Lưới thi công là mạng lưới nhiều bậc, được thành lập trong giai đoạn thiết kế kỹ thuật công trình và là cơ sở trắc địa cho việc bố trí tổng thể, bố trí chi tiết và đo vẽ hoàn công công trình. Lưới khống chế thi công có những đặc điểm sau:

- Cấu trúc hệ thống lưới được xây dựng theo nguyên tắc từ tổng thể đến cục bộ, đồng thời yêu cầu độ chính xác đối với từng bậc lưới lại tăng dần.

- Các bậc lưới thi công cần được định vị trong cùng một hệ tọa độ để bảo đảm tính thống nhất trong phân bố các hạng mục công trình.

Yêu cầu độ chính xác và đồ hình lưới được tính toán, lựa chọn trên cơ sở đáp ứng các chỉ tiêu của công tác bố trí công trình và đo vẽ hoàn công. Vì vậy, lưới thi công phải được xây dựng và xử lý số liệu theo các nguyên tắc:

- Là mạng lưới độc lập cục bộ (để tránh ảnh hưởng sai số số liệu gốc).

- Tất cả các bậc lưới thi công cần phải được tính tọa độ (độ cao) trong một hệ thống thống nhất, đã được lựa chọn trong giai đoạn khảo sát công trình.

Việc thực hiện các quy định trên đảm bảo cho lưới thi công không bị biến dạng do ảnh hưởng sai số số liệu gốc, đồng thời, tất cả các bậc lưới được định vị trong cùng một hệ tọa độ chung.

Phân tích khả năng ứng dụng các phương pháp bình sai đối với lưới thi công công trình có thể nhận thấy: phương pháp bình sai lưới phụ thuộc sẽ dẫn đến ảnh hưởng sai số số liệu gốc trong kết quả xử lý và như vậy sẽ gây ra sự biến dạng của từng bậc lưới. Phương án bình sai với sai số số liệu gốc hoặc bình sai gộp tổng thể các bậc lưới trong hệ thống là đúng về nguyên tắc nhưng khó thực hiện trong điều kiện thực tế trắc địa công trình, phương án bình sai với số liệu gốc tối thiểu (lưới tự do bậc 0 ) bảo toàn được cấu trúc nội tại của lưới nhưng có nhược điểm là thiếu chặt chẽ về phương diện định vị lưới; quy luật lan truyền sai số sẽ dẫn đến tình trạng các điểm càng xa điểm gốc sẽ có sai số tích luỹ lớn. Chỉ còn phương án bình sai lưới tự do đáp ứng được các yêu cầu đối với lưới thi công: tránh được ảnh hưởng của sai số số liệu gốc, quá trình định vị lưới được thực hiện linh hoạt, phù hợp với điều kiện thực tế.

Để định vị lưới thi công vào vị trí gần đúng nhất so với hệ tọa độ đã có trên công trình xây dựng cần chọn ma trận $\mathrm{C}$ trong công thức (3) theo quy tắc:

$$
\mathbb{C}=\left\{\begin{array}{l}
B \text { - đối với các điểm định vị } \\
0 \text { - đối với các điểm khác }
\end{array}\right.
$$


Trong công thức trên, tùy thuộc vào từng dạng lưới và điều kiện định vị mà ma trận $B$ được xác định theo một trong các biểu thức (9) hoặc (11).

\section{Thực nghiệm bình sai lưới mặt bằng thi công}

Mạng lưới thực nghiệm được chọn là lưới mặt bằng thi công tại công trình xây dựng thủy điện Sơn La. Mạng lưới có 11 điểm, bao gồm 3 điểm gốc là: PV04, PV08 và $P V 11$. Các điểm thiết kế (8 điểm) được phân bố xung quanh các hạng mục công trình đầu mối (xem hình 1). Các điểm của lưới được chọn đặt tại những vị trí thuận lợi cho việc đưa tim tuyến và đo đạc kiểm tra, hoàn công các hạng mục công trình trong quá trình thi công xây dựng.

Các thông số kỹ thuật của mạng lưới được đưa ra (xem bảng 1). Tọa độ các điểm khởi tính đưa ra (xem bảng 2). Số liệu đo góc và đo chiều dài cạnh của lưới được trình bày trong các bảng (xem bảng 3 và 4). Mạng lưới được xử lý theo phương pháp bình sai tự do bằng phần mềm chuyên dùng tại bộ môn trắc địa công trình (Trường Đại học Mỏ - Địa chất). Kết quả bình sai tọa độ thể hiện trong bảng (xem bảng 5).
Nhận xét đánh giá kết quả tính toán thực nghiệm: Kết quả tính thực nghiệm đối với lưới mặt bằng tại dự án xây dựng thủy điện Sơn La cho thấy cấu trúc nội tại của lưới không bị biến dạng do ảnh hưởng của sai số số liệu gốc, đồng thời mạng lưới được định vị tối ưu trong hệ tọa độ đã xác lập tại địa điểm xây dựng công trình.

\section{Kết luận}

1- Kết quả phân tích lý thuyết và thực nghiệm cho thấy rằng: Việc xử lý số liệu các mạng lưới thi công công trình theo phương pháp bình sai tự do là cần thiết. Phương pháp bình sai này cho phép giải quyết các mục tiêu đề ra đối với các mạng lưới mặt bằng thi công công trình.

2- Các kết quả nghiên cứu và ứng dụng thực tiễn trong thời gian dài với nhiều dạng công trình đã chứng tỏ tính đúng đắn, ưu việt của phương pháp bình sai tự do trong xử lý số liệu lưới trắc địa công trình. $\bigcirc$

\section{Tài liệu tham khảo}

[1]. Trần Khánh (1994). Nghiên cứu ứng dụng kỹ thuật vi tính trong xử lý số liệu trắc địa công trình. Báo cáo đề tài nghiên cứu khoa học cấp bộ, B91-18-01,1994, Đại học

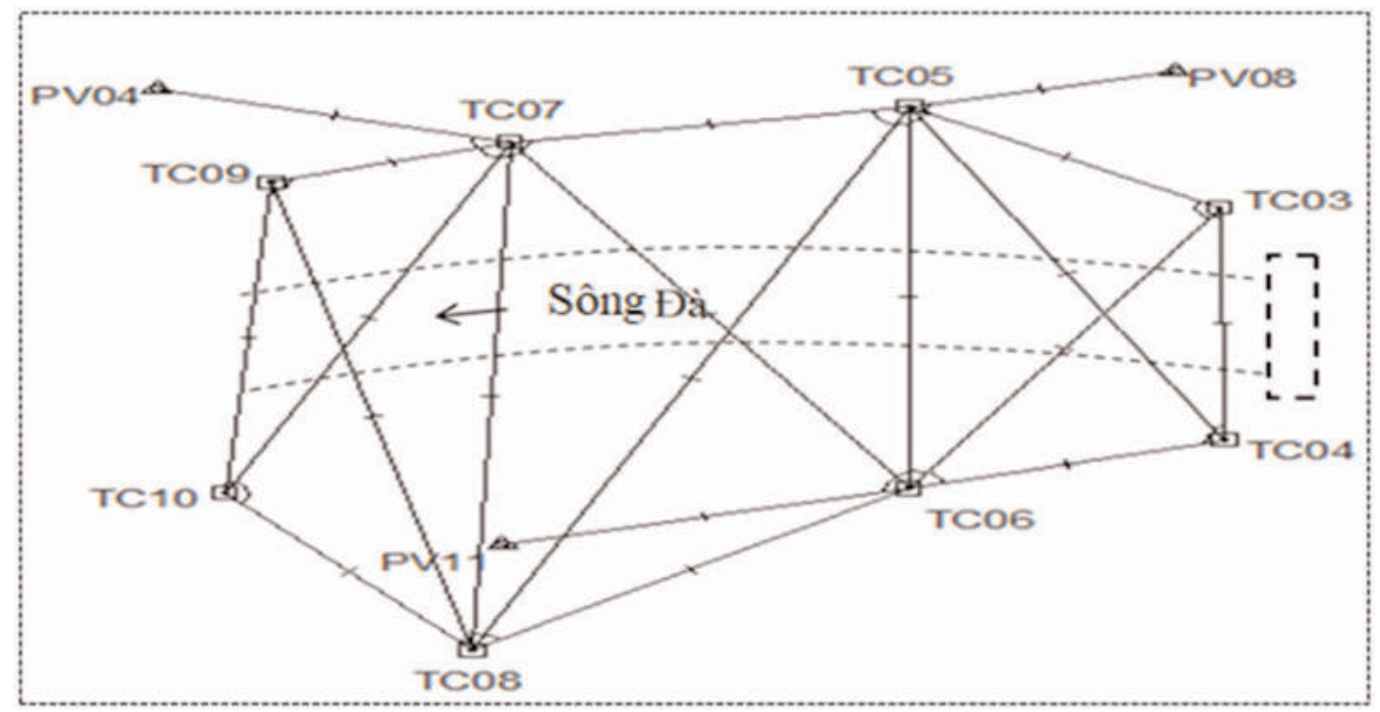

Hình 1: Sơ đồ lưới thi công thủy điện Sơn La 
Bảng 1: Thông số của mạng lưới

\begin{tabular}{|c|l|c|c|l|c|}
\hline STT & \multicolumn{1}{|c|}{ Tên tham số } & Giá trị & STT & \multicolumn{1}{|c|}{ Tên tham số } & Giá trị \\
\hline 1 & Số điểm gốc & 3 & 7 & Sai số đo góc & $1.5 "$ \\
\hline 2 & Số điểm mới lập & 8 & 8 & Sai số đo cạnh & $3+2$ ppm \\
\hline 3 & Số lượng góc đo & 27 & 9 & Hệ tọa độ & Cục bộ \\
\hline 4 & Số lượng cạnh đo & 19 & 10 & Độ cao mặt chiếu & $170(\mathrm{~m})$ \\
\hline
\end{tabular}

Bảng 2: Tọa độ các điểm khởi tính

\begin{tabular}{|c|c|c|c|c|c|}
\hline \multirow{2}{*}{ Tên điểm } & \multicolumn{2}{|c|}{ Tọa độ } & \multirow{2}{*}{ Độ cao $\mathbf{H}(\mathbf{m})$} & \multirow{2}{*}{ Cấp, Hạng } \\
\cline { 3 - 4 } & & $\mathbf{x}(\mathbf{m})$ & $\mathbf{y}(\mathbf{m})$ & & IV \\
\hline 1 & PV04 & 2378914.803 & 499183.860 & 438.0 & IV \\
\hline 2 & PV08 & 2378965.421 & 500790.981 & 296.0 & IV \\
\hline 3 & PV11 & 2377599.868 & 499729.461 & 259.0 & \\
\hline
\end{tabular}

Bảng 3: Kết quả đo góc

\begin{tabular}{|c|c|c|c|c|c|c|c|}
\hline \multicolumn{3}{|c|}{ Ký hiệu góc } & Góc đo & \multicolumn{3}{|c|}{ Ký hiệu góc } & Góc đo \\
\hline Trái & Giữa & Phải & $\left(\begin{array}{lll}0 & ,\end{array}\right)$ & Trái & Giữa & Phải & $\left(\begin{array}{lll}0 & ,\end{array}\right)$ \\
\hline TC04 & TC03 & TC06 & 315400.2 & TC05 & TC07 & TC06 & 670527.8 \\
\hline TC06 & TC03 & TC05 & 893610.7 & TC06 & TC07 & TC08 & 342746.8 \\
\hline TC06 & $\mathrm{TC} 04$ & TC05 & 782011.6 & TC08 & TC07 & TC10 & 213846.5 \\
\hline TC05 & TC04 & TC03 & 263622.6 & TC10 & TC07 & TC09 & 490253.5 \\
\hline TC08 & TC05 & TC03 & 443053.7 & TC09 & TC07 & TC04 & 322355.2 \\
\hline TC03 & TC05 & TC04 & 315325.6 & TC10 & TC08 & TC09 & 273430.9 \\
\hline TC04 & TC05 & TC06 & 272556.1 & TC09 & TC08 & TC07 & 153156.5 \\
\hline TC06 & TC05 & TC08 & 233733.5 & TC07 & TC08 & TC05 & 212534.8 \\
\hline TC08 & TC05 & TC07 & 570109.6 & TC05 & TC08 & TC06 & 320752.8 \\
\hline TC08 & TC06 & PV11 & 195827.7 & TC07 & TC09 & TC08 & 934623.7 \\
\hline PV11 & TC06 & TC07 & 720017.6 & TC08 & TC09 & TC10 & 175420.6 \\
\hline TC07 & TC06 & TC05 & 321547.7 & TC09 & TC10 & TC07 & 191622.3 \\
\hline TC05 & TC06 & TC04 & 310428.2 & TC07 & TC10 & TC08 & 1151446.3 \\
\hline TC03 & TC06 & $\mathrm{TCO} 3$ & 430925.5 & & & & \\
\hline
\end{tabular}

Mỏ địa chất.

[2].Trần Khánh (1996). Thuật toán bình sai tự do và ứng dụng trong xử lý số liệu một số mạng lưới trắc địa chuyên dùng. Tuyển tập các công trình khoa học Mỏ địa chất, số
21,1996, đại học Mỏ địa chất.

[3].Markyze.lu.M (1988) .Thuật toán và chương trình bình sai lưới trắc địa. NXB " Nhedra", Moskva-1988 (tiếng Nga). $O$ 
Bảng 4: Kết quả đo cạnh

\begin{tabular}{|c|c|c|c|c|c|c|c|}
\hline \multirow{2}{*}{ STT } & \multicolumn{2}{|c|}{ Ký hiệu cạnh } & \multirow{2}{*}{$\begin{array}{c}\text { Cạnh đo } \\
\text { (m) }\end{array}$} & \multirow{2}{*}{ STT } & \multicolumn{2}{|c|}{ Ký hiệu cạnh } & \multirow{2}{*}{$\begin{array}{l}\text { Cạnh đo } \\
\text { (m) }\end{array}$} \\
\hline & Đầu & Cuối & & & Đầu & Cuối & \\
\hline 1 & TC03 & TC04 & 670.959 & 11 & TC06 & TC08 & 830.339 \\
\hline 2 & TC03 & TC05 & 568.788 & 12 & TC06 & PV11 & 660.814 \\
\hline 3 & TC03 & TC06 & 947.767 & 13 & TC07 & TC08 & $1466 . .477$ \\
\hline 4 & TC04 & TC05 & 1082.842 & 14 & TC07 & TC09 & 393.543 \\
\hline 5 & TC04 & TC06 & 518.370 & 15 & TC07 & TC10 & 1107.968 \\
\hline 6 & TC05 & TC06 & 1101.960 & 16 & TC07 & PV04 & 579.202 \\
\hline 7 & TC05 & TC07 & 638.615 & 17 & TC08 & TC09 & 1387.020 \\
\hline 8 & TC05 & TC08 & 1712.748 & 18 & TC08 & TC10 & 598.068 \\
\hline 9 & TC05 & TC08 & 430.687 & 19 & TC09 & TC10 & 900.497 \\
\hline 10 & TC06 & TC07 & 1180.421 & & & & \\
\hline
\end{tabular}

Bảng 5: Kết quả tọa độ bình sai

\begin{tabular}{|c|c|c|c|c|c|c|}
\hline \multirow{2}{*}{} & \multirow{2}{*}{ Tên điểm } & \multicolumn{2}{|c|}{ Toạ độ } & \multicolumn{3}{c|}{ Sai số vị trí điểm $(\mathbf{m})$} \\
\cline { 3 - 7 } & & $\mathbf{X}(\mathbf{m})$ & $\mathbf{Y}(\mathbf{m})$ & $\mathbf{M x}$ & $\mathbf{M y}$ & $\mathbf{M p}$ \\
\hline 1 & TC03 & 2378572.0732 & 500861.1327 & 0.0017 & 0.0018 & 0.0025 \\
\hline 2 & TC04 & 2377901.1696 & 500869.3738 & 0.0019 & 0.0019 & 0.0027 \\
\hline 3 & TC05 & 2378863.3110 & 500372.5657 & 0.0015 & 0.0015 & 0.0021 \\
\hline 4 & TC06 & 2377761.3664 & 500370.2188 & 0.0016 & 0.0016 & 0.0022 \\
\hline 5 & TC07 & 2378760.8513 & 499742.2318 & 0.0015 & 50.001 & 0.0021 \\
\hline 6 & TC08 & 2377295.6091 & 499682.8306 & 0.0016 & 0.0015 & 0.0030 \\
\hline 7 & TC09 & 2378645.8966 & 499365.8581 & 0.0015 & 0.0010 & 0.0025 \\
\hline 8 & TC10 & 2377748.4323 & 499292.1564 & 0.0019 & 0.0012 & 0.0030 \\
\hline 9 & PV04 & 2378914.7997 & 499183.8806 & 0.0015 & 0.0018 & 0.0023 \\
\hline 10 & PV08 & 2378965.4259 & 500790.9662 & 0.0012 & 0.0018 & 0.0022 \\
\hline 11 & PV11 & 2377599.8664 & 499729.4551 & 0.0016 & 0.0013 & 0.0020 \\
\hline
\end{tabular}

\section{Summary}

\section{Application of free network adjustment in processing of the hozizotal setting out control}

Nguyen Ha, Tran Thuy Linh

University of Mining and Geology

The content of the newspaper is about the application of free adjustmet in data processing construction networks. In the newspaper we also proposed the algorithm and the suitable calculation prosess for this construction type of networks. Through the study on theory and the results that we collected from production in reality, we assert that the free adjustment is the superiority method that can be aplied to process data of many kinds of geodetic networks. O 